\title{
Une hiérarchie privative pour le français
}

\author{
Tifrit, Ali, \& Voeltzel, Laurence \\ Laboratoire de Linguistique EA 3827 / Université de Nantes \\ \{Ali.Tifrit et Laurence.Voeltzel\}@univ-nantes.fr
}

\section{Introduction}

Nous defendons l'hypothèse qu'une représentation unaire des obstruantes du français peut intégrer l'hypothèse contrastiviste telle qu'elle est appliquée dans les cadres binaristes ${ }^{1}$. Nous montrons que, sur la base de la phénoménologie diachronique du français ${ }^{2}$, seule l'utilisation de primitives privatives permet d'expliquer et de justifier les mécanismes ciblant les obstruantes. Cette proposition constitue une révision majeure des représentations classiques du français. Elle a aussi pour conséquence de reconsidérer l'utilisation des éléments : nous montrons qu'ils ne constituent pas une traduction des traits.

Le présent article est organisé de la manière suivante. Nous présentons tout d'abord le cadre contrastiviste tel qu'il est défini par Dresher (2009), Dresher et al. (2013), Hall (2007). Nous explicitons la démarche qu'ils adoptent et qui s'oppose à la méthode des paires minimales. Nous pointons les avantages du cadre contrastiviste, notamment, parce que représentations et processus sont associés.

Nous abordons ensuite le cas des obstruantes du français et les structurations qui en ont été proposées. Elles s'inscrivent dans deux perspectives : linéaire ou tridimensionnelle. Ces approches présentent toutes deux des limites, lesquelles ne peuvent être dépassées sans intégration de la dimension syllabique ${ }^{3}$ et de ses conséquences sur le contenu segmental.

Nous nous tournons enfin vers une représentation élémentaire qui dépasse les apories des modèles précédemment cités. Le comportement des obstruantes permet de construire une structure intégrant un contraste privatif.

\section{Approche contrastive}

La Hiérarchie Contrastive (Dresher, 2009) offre un moyen de représenter les systèmes phonémiques non pas en se basant sur une description phonétique des segments, mais en s'appuyant sur leur fonction au sein du système.

Les segments contiennent deux types d'informations, encodées, selon l'approche classique, sous la forme de traits binaires : des informations phonétiques (articulatoires et perceptives) et des informations phonologiques. Alors que le premier type est requis pour fournir à la forme sous-jacente une réalisation effective, le second type sert à distinguer quels segments sont impliqués ou non dans des processus phonologiques donnés (Hall, 2007 : 20). Ainsi, sur la totalité des informations, ou traits, contenus dans un segment, seuls certains d'entre eux sont utilisés par la composante phonologique. Comment identifier ces traits? En suivant l'Hypothèse Contrastiviste (Hall, 2007), on considèrera que seuls les traits nécessaires à la distinction des phonèmes d'une langue sont phonologiquement actifs - c'est-à-dire impliqués dans des processus phonologiques. On nomme ces traits contrastifs puisqu'ils permettent de distinguer les phonèmes entre eux au sein d'un système et donc, de les faire contraster. Dans le contenu segmental, ce qui n'est pas contrastif est alors dit redondant. On considèrera également ces traits comme passifs, autrement dit inactifs dans l'activité phonologique de la langue à laquelle ils appartiennent.

\subsection{Structuration des traits}

La méthode dite du Successive Division Algorithm (SDA; Dresher, 2009) s'avère particulièrement efficace pour accéder directement aux traits contrastifs d'une langue et éviter les traits redondants ${ }^{4}$. 
$S D A$ découle de l'approche de Jakobson \& Lotz (1949) et de leur analyse de l'organisation interne des segments du français (sur laquelle nous reviendrons dans la partie suivante). La méthode, explicitée par Dresher (2009 : 22 - 25), consiste à organiser les traits distinctifs de telle sorte que seuls les traits contrastifs apparaissent dans la structure hiérarchique finale. Pour y parvenir, on distingue trois étapes $(2009: 25)$ :

- Premièrement, considérer tous les phonèmes de l'inventaire étudié comme les allophones d'un seul phonème, sans y chercher de spécifications de traits en particulier.

- Deuxièmement, repérer au moins un membre qui se distingue des autres et utiliser un trait distinctif qui permet de diviser l'ensemble en autant de sous-ensembles possibles (les branchements sont toujours binaires, à savoir un groupe $[+\mathrm{T}]$ et un groupe $[-\mathrm{T}]$ où $\mathrm{T}$ est un trait pertinent).

- Troisièmement, répéter la seconde étape dans tous les sous-ensembles jusqu'à ce que chaque segment de l'inventaire soit spécifié pour un trait.

Si l'on prend l'exemple d'un inventaire simple, tel que $/ \mathrm{t}, \mathrm{d}, \mathrm{n} /$, on peut envisager deux manières de l'organiser hiérarchiquement. On peut tout d'abord opérer dans l'ensemble $/ \mathrm{t}, \mathrm{d}, \mathrm{n} / \mathrm{une}$ première distinction au niveau de la nasalité et isoler /n/ du reste. Ensuite, /t/ et /d/ se distinguent uniquement par le voisement, ce qui constitue le second trait contrastif de l'inventaire. On peut alors le représenter de la manière suivante, en Figure 1 (a) :

Figure 1 : Les deux organisations hiérarchiques possibles de /t, d, n/

(a)

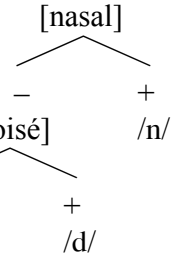

(b)

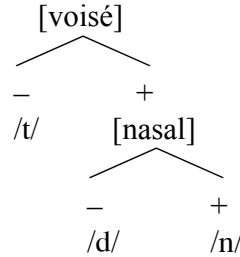

Il est par ailleurs possible d'organiser les deux traits contrastifs précédemment déduits dans un ordre différent ; on peut tout à fait proposer une partition de l'inventaire /t, $\mathrm{d}, \mathrm{n} / \mathrm{en}$ sélectionnant le contraste de voisement avant le contraste de nasalité, voir Fig.1 (b).

Alors que les deux hiérarchies présentées en Fig. 1 exploitent les mêmes traits, les segments ne sont pas spécifiés de la même manière. Dans le premier cas, si l'on place le résultat de la hiérarchie (a) dans un tableau on obtient le Tableau 1 (a). Le résultat de la hiérarchie en (b) est illustré dans le Tableau 1 (b).

Tableau 1 : Traduction des hiérarchies de la Fig. 1 en matrices

\begin{tabular}{l|c|c|c} 
& $/ \mathrm{t} /$ & $/ \mathrm{d} /$ & $/ \mathrm{n} /$ \\
\hline [voisé & - & + & \\
[nasal] & - & - & +
\end{tabular}

(b)

\begin{tabular}{l|c|c|c} 
& $/ \mathrm{t} /$ & $/ \mathrm{d} /$ & $/ \mathrm{n} /$ \\
\hline [voisé] & - & + & + \\
[nasal] & & - & +
\end{tabular}

Dans les deux cas, la hiérarchie permet de révéler les contrastes entre tous les phonèmes du système. En effet, la structuration hiérarchique impose que chaque segment soit associé à une valeur de trait et isolé des autres segments. 


\subsection{Le Pairwise Algorithm}

$\mathrm{Si}$, en revanche, l'on établit une répartition, comme celle illustrée en Tableau 2, c'est-à-dire sans considérer d'organisation hiérarchique et par simple observation du système, nous spécifions entièrement chaque segment en fonction des deux traits utiles à leur caractérisation :

Tableau 2 : Spécifications dans le Pairwise Algorithm

\begin{tabular}{l|c|c|c} 
& $/ \mathrm{t} /$ & $\mathrm{d} /$ & $\mathrm{ln} /$ \\
\hline [voisé] & - & + & + \\
[nasal] & - & - & +
\end{tabular}

Afin d'élucider les relations de contraste, il est ensuite nécessaire de supprimer tous les traits jugés redondants (cases grises). Le premier trait à disparaître aurait alors été le trait logiquement redondant [+voisé] dans le cas d'un segment spécifié [+nasal], puisque une nasale est par défaut voisée. Le second trait redondant, [-voisé] pour /p/, doit aussi disparaître, puisque, selon la même logique, un segment qui n'est pas voisé, ne peut être nasal.

La représentation dans le Tableau 2 est le résultat obtenu après application du Pairwise Algorithm (Dresher 2009 : 20) à notre inventaire /t, d, n/. Le PA spécifie complètement le système pour ensuite y soustraire les traits redondants. Cette méthode peut donner l'impression de ne conserver que les traits strictement utiles à la caractérisation des contrastes et de s'être débarrassé des traits superflus. Cependant, le choix de ces traits « inutiles » est entièrement déterminé par l'observateur et ne semble pas s'imposer de lui-même. Ce côté arbitraire dans l'établissement des relations de contraste peut amener à négliger certaines paires minimales : en 3 , une fois les traits redondants supprimés, $/ \mathrm{t} / \mathrm{et} / \mathrm{n} / \mathrm{ne}$ partagent aucun trait phonologique et ne peuvent pas contraster. Si on veut décrire la relation entre $/ \mathrm{t} / \mathrm{et} / \mathrm{n} /$, il faut réintroduire un trait jugé précédemment inutile (soit le trait [-nasal] pour $/ \mathrm{t} /$, soit le trait [+voisé] pour $/ \mathrm{n} /$ ), ce qui remet en question l'efficacité de cette méthode PA.

\subsection{Processus}

La méthode SDA, si elle contourne cette difficulté en évitant le côté trop arbitraire du choix des traits à supprimer, présente toutefois des limites. Comme nous l'avons vu, l'ordre considéré permet de décrire plusieurs hiérarchies, de fait, surgénérant des contrastes. Le SDA permet de conserver les traits nécessaires à la distinction des phonèmes mais rien ne permet de dériver l'ordre dans lequel ils doivent apparaitre dans la hiérarchie. La portée attribuée aux traits dépend, ici aussi, de l'auteur qui sélectionne une hiérarchie particulière, cependant, en accord avec les processus observés dans une langue donnée.

Le hawaïen présente le système à 8 consonnes suivant, en (1) (voir Elbert \& Pukui, 1979) :

(1) Le système consonantique du hawaïen

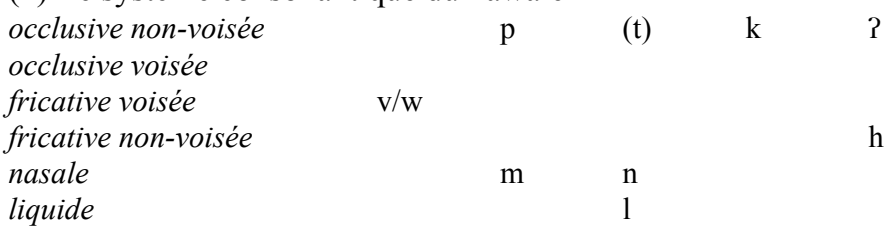

Pour caractériser ce système, quatre traits sont nécessaires [sonorant], [labial], [constricted glottis], [spread glottis] (Clements, $2003: 290$ ). Les hiérarchies possibles $\left(4^{4}\right)$ sont cependant réductibles si l'on considère le comportement dans les emprunts à l'anglais, voir le corpus en (2) :

(2) Emprunts à l'anglais laitue : lettuce /lekuke/

savon : soap /kope/

bière : beer /pia/

douzaine : dozen /kaakini/

brosse : brush /palaki/ machine : machine /mikini/ ticket : ticket /kikiki/

Norvège : Norway/nolewai/

velours : velvet /welaweka/

On remarque dans le corpus précédent que les obstruantes coronales sont adaptées en /k/. Plus important, en dépit de la présence de la fricative glottale, même les fricatives sont interprétées comme des 
obstruantes vélaires alors que /h/ semble désigné pour s'y substituer. En hawaïen, les fricatives sont assimilées à /k/. Pourquoi ? Vraisemblablement, la fricative glottale ainsi que la vélaire occupent une place particulière dans la hiérarchie du hawaïen qui empêche ou autorise l'adapation.

La hiérarchie suivante, en Fig. 2, implémente ces contraintes. Elle rend compte du fait que /k/ est le segment le moins spécifié de la langue (tous ses traits sont valués négativement) et joue le rôle d'une consonne par défaut. En revanche, /h/ qui est spécifié pour les traits de dimension glottale acquiert un rôle spécifique dans le système qui ne lui permet pas de remplir une autre fonction, de remplacer des segments inconnus du système.

Figure 2 : Hiérarchie contrastive du hawaïen

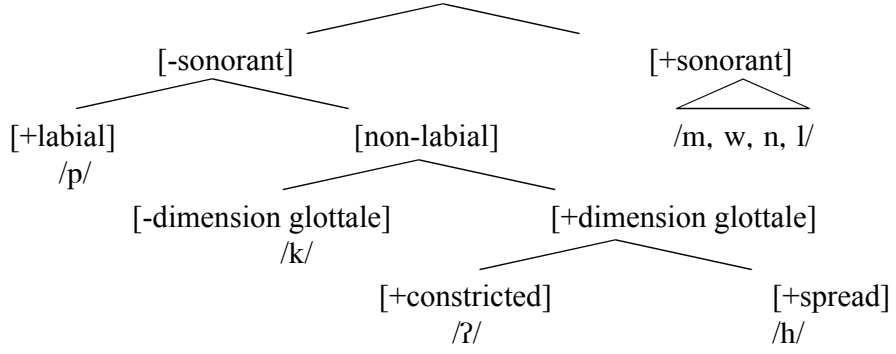

En d'autres termes, l'hypothèse contrastiviste, pour être menée à bon terme, ne peut être envisagée en dehors de l'exploration des processus phonologiques.

Nous nous tournons à présent vers le cas du français pour lequel nous appliquons cette démarche à partir de deux approches structurales qui se distinguent par leur vision de ce système consonantique.

\section{Deux représentations concurrentes pour le français}

Selon les hypothéses défendues, ouvertement ou non, le système des obstruantes du français peut être représenté de deux façons : une représentation où chaque point d'articulation constitue une propriété distinctive qui s'oppose à une représentation où ce qui compte est l'unité comportementale, la convergence en termes de processus.

\subsection{Linéaire vs tridimensionnelle}

Le premier type est représenté par Martinet (1970) où, puisque la catégorie occlusive ne comporte pas de correspondant fricatif direct, c'est-à-dire qui s'articulerait «précisément ») au même point, chaque paire de segment est représentée le long d'un axe qui modélise l'espace compris entre les lèvres et le voile du palais 7(a). La seconde option, celle tenue par Jakobson (1984), considère que la cavité buccale, loin d'être un continuum, dessine deux types de cavités (longue et divisée) dont l'orientation permet de décrire quatre catégories (longue point de constriction à l'avant, longue point de constriction à l'arrière, divisée point de constriction à l'avant, divisée point de constriction à l'arrière) :

(3) Division de la cavité buccale et lieux d'articulation.

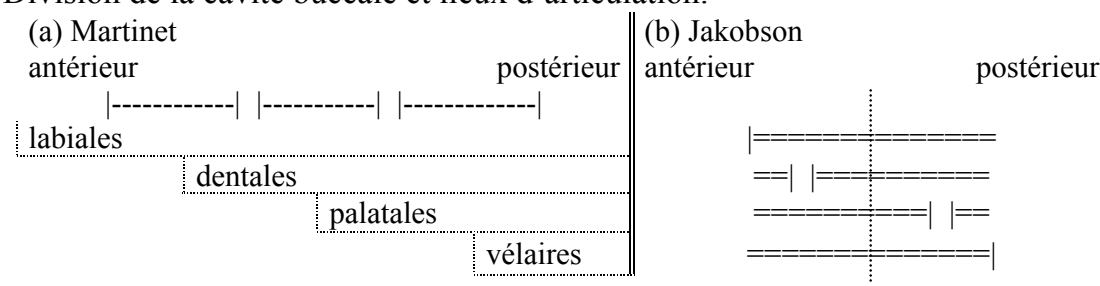

Légende : ||$=$ point de constriction ; ----- : continuum palatal (dent $>$ voile) ; === : résonateur. La ligne pointillée verticale symbolise la symétrie des ensembles labiales/dentales et palatales/dorsales; on notera par ailleurs la symétrie labiales/vélaires et dentales/palatales. 
Ces deux modes de représentation prédisent deux structurations différentes du système qui découlent des procédures de découvertes précédemment illustrées : PA vs SDA. Ces deux positions antagonistes ne reposent pas sur la nature acoustique ${ }^{5}$ vs articulatoire de la description mais sur le système de traits manipulés : analogique dans un cas, strictement binaire dans l'autre.

Dans le cadre martinetien, il ne saurait y avoir de rapport direct entre les occlusives labiales et les fricatives labiodentales. Ces deux ensembles, situés en des points d'articulation différents, ne peuvent en aucun cas être alignés sur une même dimension [labial $]^{6}$. Les consonnes s'organisent donc linéairement : le mode, $[ \pm$ continu], contraint cette représentation. En effet, pour chaque distinction occlusif/fricatif il y a déplacement du point d'articulation de telle sorte que les fricatives sont nécessairement séparées des occlusives correspondantes. Ce qui permet de reconduire l'une des corrélations constitutives du français : la rapport proportionnel de voisement (Martinet, $1970: 73$ ).

Tableau 3 : Représentation linéaire du système des obstruantes en français

\begin{tabular}{c|c|c|c|c|c|c} 
& labial & labio-dental & dental & alvéolaire & palatal & vélaire \\
\hline non-voisé & $\mathrm{p}$ & $\mathrm{f}$ & $\mathrm{t}$ & $\mathrm{s}$ & $\int$ & $\mathrm{k}$ \\
voisé & $\mathrm{b}$ & $\mathrm{v}$ & $\mathrm{d}$ & $\mathrm{z}$ & 3 & $\mathrm{~g}$
\end{tabular}

Dans le cas de la représentation tridimensionnelle appliquée au français par Jakobson et Lotz (1949), occlusives et fricatives sont systématiquement associées. En effet, par symétrie vis-à-vis d'un axe vertical (en pointillés en (3)(b)), on peut associer dentales et labiales (antérieures), d'un côté, et palatales et vélaires (postérieures), de l'autre, de même les couples [labiales / vélaires] et [palatales / dentales] sont associables parce qu'ils partagent une configuration de résonateur en image-miroir (respectivement indivis et divisé, la relation est symbolisée par des flèches en (3)(b) précédente). Dans ce cadre, la distinction occlusif/fricatif est secondaire. La géométrie du résonateur est, elle, décisive : la PERCEPTIBILITÉ\{compact,diffus\} détermine le reste de la structure. Ainsi, TONALITÉ \{grave,aigu et QUALITÉ \{mat,strident\} sont plus bas dans la hiérarchie comme l'illustre le Tableau 4 suivant :

Tableau 4 : Organisation du système selon Jakobson

\begin{tabular}{|c|c|c|c|c|}
\hline & \multicolumn{3}{|c|}{ PERCEPTIBILITÉ } \\
\hline & & \multicolumn{2}{|c|}{ diffus } & compact \\
\hline & & \multicolumn{2}{|c|}{ TONALITÉ } & \multirow{4}{*}{$\begin{array}{l}\mathrm{k} / \mathrm{g} \\
\int / 3\end{array}$} \\
\hline & & & grave & \\
\hline \multirow{2}{*}{ QUALITÉ } & mat & $\mathrm{t} / \mathrm{d}$ & \multirow{2}{*}{$\begin{array}{l}\mathrm{p} / \mathrm{b} \\
\mathrm{f} / \mathrm{v}\end{array}$} & \\
\hline & strident & $\mathrm{s} / \mathrm{z}$ & & \\
\hline
\end{tabular}

La raison de cette structuration des traits est double. D'une part, dans ce système, et contrairement au cadre précédent, consonnes et voyelles partagent les mêmes traits. D'autre part, cette structuration fait écho aux travaux concernant l'acquisition de la phonologie. Pour Jakobson (1969), le première stade de l'acquisition émerge du contraste, à l'intérieur d'une syllabe CV non-marquée, entre une voyelle-noyau associée au trait de PERCEPTIBILITÉ \{majeur\}, [compact], et une consonne [diffuse]. La hiérarchie contrastive doit en conséquence se conformer à la stratification du système dans l'acquisition et la bifurcation de TONALITÉ n'est possible qu'une fois que celle de PERCEPTIBILITE est acquise.

\subsection{Fricatives}

Autrement dit, l'ordre des traits dans la structure, PERCEPTIBILITÉ \{compact,diffus\} >> TONALITÉ \{grave,aigu $\}>$ QUALITÉ \{mat,strident\}, correspond à l'ordre dans lequel ils sont acquis : 
Figure 3 : La hiérarchie contrastive des obstruantes du français

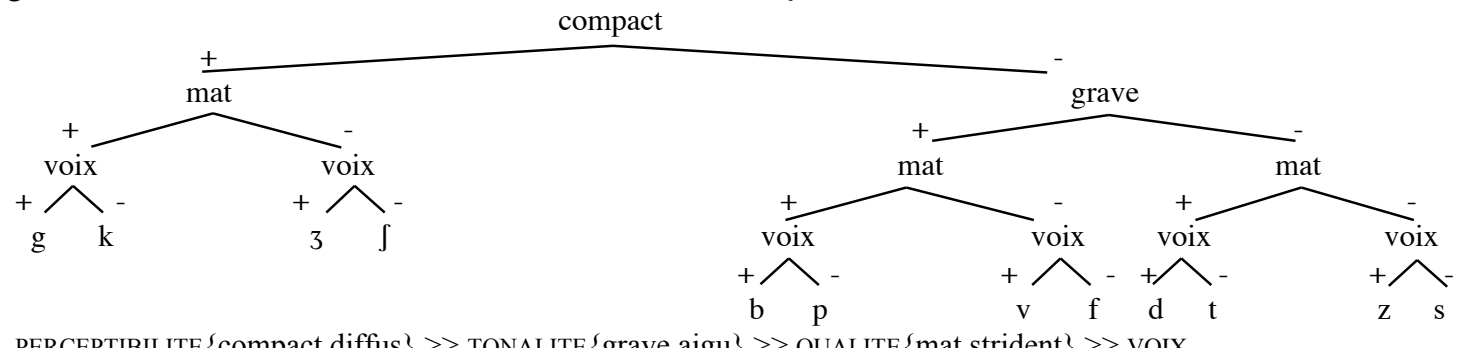

PERCEPTIBILITE $\{$ compact,diffus $\}>>$ TONALITE $\{$ grave,aigu $\} \gg$ QUALITE $\{$ mat,strident $\}>$ vOIX

On aura noté que rien dans le cadre défini par Jakobson ne permet de justifier la place qu'occupent les fricatives palatales dans la représentation en Fig. 3 précédente. En effet, le contraste de TONALITÉ \{aigugrave\} devrait aussi s'appliquer aux segments compacts ce qui nous mènerait à un déséquilibre dans la structure où les segments [+compact, +grave] seraient automatiquement occlusifs et où l'association [+compact, -grave] définirait des segments fricatifs. Autrement dit, où TONALITÉ devrait aussi s'appliquer, générant des segments inattendus ou des places inoccupées (en gras dans la Fig. 4 suivante) dans la structure.

Figure 4 : Application de TONALITÉ aux segments [+compact]

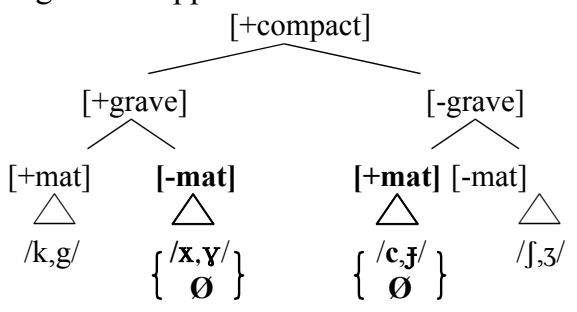

Jakobson (1984:41-42) y apporte une réponse partielle :

En français, aucune consonne continue n'a exactement le même point d'articulation qu'une occlusive ; cette différence est évidemment due à ce que dans les continues optimales la friction et la turbulence sont bien plus importantes que dans les occlusives optimales.[...] Le bruit plus intense produit par les obstruantes stridentes requiert une barrière plus dure. C'est pourquoi, outre les lèvres, seul instrument utilisé dans les bi-labiales, les labio-dentales emploient aussi les dents, tandis que les sifflantes mobilisent les dents inférieures, en plus des obstacles employés pour les non-stridentes.[...] Et si les obstruantes compactes ne présentent pas d'opposition grave/aigu, les occlusives $/ \mathrm{k} /$ et $/ \mathrm{g} /$ trouvent une contrepartie stridente dans les sifflantes compactes $/ \mathrm{J} / \mathrm{et} / 3 /$. En français, les trois types de fricatives requièrent l'utilisation des dents comme obstacle supplémentaire.

Jakobson admet donc, à l'instar de la position de Martinet, qu'il n'y a nulle part en français de correspondance exacte occlusif / fricatif. Cependant, il pointe le fait que les segments compacts n'entrent pas dans un rapport de TONALITÉ. Loin d'un simple renvoi à une dimension phonétique, où l'obstacle rencontré, les dents, augmente la fréquence du bruit, une forme d'enhancement ${ }^{7}$, c'est la relation entre les unités qui importe ici.

Les rapports au sein des obstruantes sont proportionnels : $/ \mathrm{k} /$ est à $/ \mathrm{J} /$ ce que $/ \mathrm{p} /$ est à $/ \mathrm{f} /$ et $/ \mathrm{t} / \mathrm{à} / \mathrm{s} /$. Dans les termes de Troubetzkoy (1939), ils constituent des oppositions bilatérales privatives opposant un être marqué à sa contrepartie non marquée. Ce qui, à nouveau, est congruent avec le modèle stratificationnel de l'acquisition. Dans le cas où la TONALITÉ s'appliquerait aux segments [+compact], il y aurait une place vide sous la bifurcation [-grave] et un segment nul [+mat] serait, par conséquent, en mesure de générer un segment plein strident. Nous reviendrons par la suite, dans la partie 4 , sur le cas des fricatives palatales et nous montrerons comment il est possible de justifier cet alignement sur les occlusives vélaires sous réserve de dépasser certaines apories du modèle. 


\subsection{Limites}

Des deux méthodes établies jusque-là pour désigner les traits contrastifs d'une langue, la méthode SDA apparaît comme la plus efficace : en effet, la méthode PA présente dès le début de son application une faiblesse en supprimant des traits jugés redondants, au risque de les réintroduire par la suite et sous peine de ne pouvoir distinguer toutes les paires possibles. La méthode SDA, en revanche, permet de conserver tous, et seulement, les traits nécessaires aux contrastes. Cependant, les résultats obtenus à partir de cette méthode peuvent grandement varier puisque, rien, lors de l'application du SDA, ne permet de contraindre l'odre d'apparition des traits dans la structure hiérarchique, ni leur surapplication.

C'est alors à l'extérieur de l'approche SDA qu'il faut regarder pour trouver une manière de supprimer le côté arbitraire de l'organisation des traits. L'économie (Clements, 2003). constitue un des moyens de sélectionner l'un des résultats générés et repose sur une forme de rentabilisation des traits. La hiérarchie correspondant à la représentation d'un système le plus économique possible est à privilégier. Une autre possibilité est de renvoyer, comme nous l'avons souligné précédemment, au schéma acquisitionnel où la sélection de la hiérarchie s'appuie sur le parallèle entre ordre stratificationnel et séparation des sousensemble d'obstruantes.

Dans le cadre du français, les deux représentations proposées peuvent apparaître déficientes pour une raison majeure : aucune mention n'est faite des conditions syllabiques permettant l'expression des segments. C'est l'absence de cette dimension, dès l'établissement de la hiérarchie, qui interdit de se défaire de cette saveur arbitraire ${ }^{8}$. En effet, rien dans la hiérarchie ne permet de voir ou de prédire les processus affectant les obstruantes, rien ne permet d'en comprendre le déclenchement. La hiérarchie seule ne fait qu'enregistrer les résultats d'une analyse inachevée qui se concentre uniquement sur le niveau segmental.

\section{Hiérarchie unaire}

\subsection{Diachronie}

Afin de justifier la représentation interne des segments, nous nous tournons vers la diachronie du français qui nous éclaire sur leur contenu. Ce détour est aussi un moyen d'intégrer la dimension syllabique notamment parce que la compréhension du changement implique de faire référence à la position occupée par les segments. Si, selon Bourciez \& Bourciez (1967 : 122), « [p]our une consonne, la position la plus forte consiste à se trouver soit à l'initiale du mot, soit à l'intérieur derrière une autre consonne ", on s'attend à ce que les positions faibles (intervocalique, préconsonantique et finale) soient le lieu de lénition permettant d'accéder à la structure interne. Si pour Scheer \& Ségéral (2001 : 121) :

\footnotetext{
[...] les lénitions ne résultent pas d'une transmission de primitives phonologiques et n'ont donc rien à voir avec l'action phonétique d'un segment adjacent. Elles renvoient à la position des segments dans la chaîne, et à cela seulement.
}

alors le contenu des segments y est directement accessible et, dans ce cas, doit permettre de voir le rappport entre segments [mat] et [strident], en particulier le rapport entre occlusives vélaires et fricatives palatales.

Les trois sites de lénition ${ }^{9}\left\{V_{-} V_{2}, C, \#\right\}$ sont relativement transparents pour les labiales et les coronales. Les occlusives se résolvent principalement en fricatives sans modification du lieu (Tableau 5 suivant). 
Tableau 5 : Lénition des labiales et des dentales

\begin{tabular}{|c|c|c|c|c|c|c|}
\hline & \multicolumn{3}{|c|}{ Labiales } & \multicolumn{3}{|c|}{ Coronales } \\
\hline & $\mathrm{V} \_\mathrm{V}$ & $-\mathrm{C}$ & \# & $\mathrm{V}-\mathrm{V}$ & $\mathrm{C}$ & _\# \\
\hline \multirow{2}{*}{ 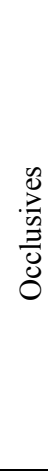 } & $\begin{array}{c}\text { habere }>\mathrm{I}^{\mathrm{er}} \text { s. haßere }> \\
\text { III }^{\text {ème2 }} \text { s. avere }> \\
\text { XVIII }^{\text {eme2 }} \text { s avoir }\end{array}$ & & $\begin{array}{c}\text { plumbu(m) } \\
>\text { plombo }> \\
\text { VII }^{\text {eme }} \text { s. } \\
\text { plomp }> \\
\text { XVII }^{\text {eme }} \text { s. } \\
\text { plomb }\end{array}$ & 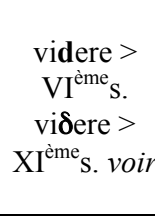 & $\begin{array}{c}\text { advenire }> \\
\text { avenir }\end{array}$ & $\begin{array}{c}\text { grande }(\mathrm{m}) \\
>\text { grande }^{\prime}> \\
\text { VII }^{\text {eme }} \mathrm{s} . \\
\text { grant }> \\
\text { XVII }^{\text {ème }} \mathrm{s} . \\
\text { grand }\end{array}$ \\
\hline & $\begin{array}{c}\text { ripa }>\mathrm{IV}_{\text {ème2 }}^{\text {è }} \text { s. riba }> \\
\mathrm{V}_{\text {ème }}^{\text {s. rißa, riva }>} \\
\mathrm{XVII}^{\text {ème2}} \text { s. rive }\end{array}$ & $\begin{array}{c}\text { rupta }> \\
\text { VII }^{\text {ème }} \mathrm{s} . \\
\text { rofte } \\
\mathrm{XVII}^{\text {ème2 }} \mathrm{s} \text {. } \\
\text { route }\end{array}$ & 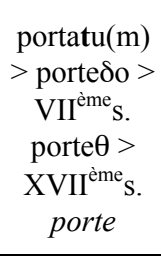 & 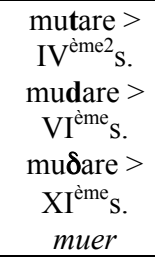 & $\begin{array}{l}\text { plat(a)nu }> \\
\text { plane }\end{array}$ & $\begin{array}{c}\text { portatu }(\mathrm{m}) \\
>\text { portedo }> \\
\text { VII }^{\text {eme }} \mathrm{s} . \\
\text { porte }>> \\
\text { XVII }^{\text {ème }} \text { s. } \\
\text { porte }\end{array}$ \\
\hline \multirow[b]{2}{*}{ 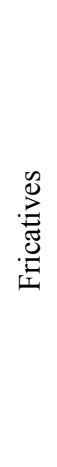 } & $\begin{array}{c}\text { lavare }>\text { laver, } \\
\text { avunculu }>\text { oncle }\end{array}$ & $\begin{array}{c}\text { nav(i)gare } \\
>\text { nager } \\
\text { viv(e)re }> \\
\text { vivre } \\
\end{array}$ & $\begin{array}{c}\text { nave }(\mathrm{m})> \\
\text { neve }> \\
\text { VII }^{\text {eme }} \text { s. nef } \\
>\text { nef }\end{array}$ & & & \\
\hline & $\begin{array}{l}\text { refusare }>\text { a.fr. reuser }> \\
\text { ruser, *malifatius }> \\
* \text { mal(i)vatiu }>\text { mauvais }\end{array}$ & $\begin{array}{c}\text { lat. } \\
\text { forf(i)ces > } \\
\text { forces, } \\
\text { *antef }(\mathrm{o}) \text { na } \\
>\text { antienne } \\
\text { *trif }(\mathrm{o}) \mathrm{lu}> \\
\text { trèfle }\end{array}$ & & $\mathrm{s}>\mathrm{z}^{10}$ & $\begin{array}{c}\text { krista }> \\
\text { XII }{ }^{\text {eme }} \text { s. krete } \\
>\text { crête, } \\
\text { responsa }> \\
\text { a.fr. response } \\
>\text { réponse, } \\
\text { musca }>\text { a.fr. } \\
\text { mosche }> \\
\text { mouche, }\end{array}$ & $\begin{array}{c}\text { clausu(m) } \\
>[\text { klozo }]> \\
\text { VII }^{\text {eme }} \mathrm{S} . \\
{[\text { klos }]>} \\
\text { clos }\end{array}$ \\
\hline
\end{tabular}

En revanche, les occlusives vélaires aboutissent à :

- des fricatives palatales : lat. carru $>$ fr. char, lat. caru $>$ fr. cher, lat. caballu $>$ fr. cheval, lat. calidu $>$ fr. chaud, lat. galbinu $>$ fr. jaune, lat. gamba $>$ fr. jambe, lat. gaudia $>$ fr. joie, lat. planca $>$ planche, lat. furca $>$ fourche, lat. bucca $>$ bouche, lat. larga $>$ large, lat. misinga $>$ mésange

- à jod ou à la semi-voyelle dorso-vélaire par résolution vocalique (après spirantisation) : fakta >

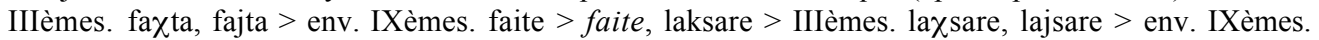
laisier $>$ laisser, lat. lacte, noctem, coxa, frax(i)nu $>$ fr. lait, nuit, cuisse, (a.fr. fraisne $>$ ) frêne, securum $>$ IVème2s. seguru $>$ Vèmes. seүuru, sewuru, seuro $>$ sûr, legem $>$ IIIèmes. lejje $>$ VIIèmes. lej $>l o i$

- la fricative coronale : placere $>$ IIIèmes. plakJere, plajtsere $>$ IVème2s. plajdzere $>$ VIIèmes. plaidzir $>$ plaisir.

- Puisqu'il n'y a pas de groupes */kp,gb/ latin, on peut signaler le destin du groupe /gm/ qui, dans un premier temps, se réduit à $/ \mathrm{w} />/ \mathrm{u} /$ et qui devient le second élément de la diphtongue dont le premier élément est constitué de la voyelle précédente (Bourciez \& Bourciez, 1967 : 144) : lat. sagma $>$ a.fr. some $>$ somme, lat. fantagma $(\mathrm{cl}$. phantasma) $>$ fantôme, lat. pigmentu $>$ a.fr. piumentu $>$ piment, lat. phlegma $>$ a.fr. fleume $>$ flegme

Il faut aussi souligner ces cas fascinants de fusion ou la labiale associée à jod produit une fricative palatale : lat. sapiam $>$ sache, lat. appropiare $>$ approcher, lat. clippiacu $>$ clichy, lat. tibia $>$ tige, lat. gubia $>$ gouge, lat. rabia $>$ rage, lat. rubeu $>$ rouge, lat. simiu $>$ singe, lat. vindemia $>$ vendange, lat. somniare $>$ songer, lat. commeatu $>$ congé, lat. cavea $>$ cage, lat. salvia $>$ sauge, lat. alveu $>$ auge. Ce qui n'est pas le cas lorsque l'on a une occlusive coronale (lat. cantione $>$ chanson, lat. fortia $>$ force, lat. infantia $>$ enfance, lat. cymatia $>$ cimaise, lat. ratione $>$ raison).

Enfin, en sortant du domaine strictement français, l'occlusive vélaire du latin en position préconsonantique aboutit à une occlusive labiale en roumain : 


\begin{abstract}
Un phénomène très controversé de la phonétique historique du roumain, à savoir le traitement des vélaires dans les groupes latins $/ \mathrm{kt}, \mathrm{ks} />/ \mathrm{pt}$,(p)s/ (lat. noctem $>$ roum. noapte, lat. pectus $>$ roum. piept, lat. coxa $>$ roum. koaps $\Lambda$, lat. frixit $>$ roum. fripse), peut être rattaché aux transformations qui ont eu lieu en finale de syllabe" (Sala, $1970: 24)$.
\end{abstract}

Ainsi, les formes latines fraxinus, infixi, factus, toxicum aboutissent en roumain à frapsin, infipse, fapta, toapsec respectivement. Un cas similaire touche aussi certaines formes dialectales du roumain où les groupes $/ \mathrm{kn} /$ sont réalisés $/ \mathrm{mn} /$ : roum. ciocni $>$ roum. dial. ciomni "heurter", roum. ocnv $>$ roum. dial. omn $\Lambda$ "saline", roum. pocne te $>$ roum. dial. pomne $\int$ te "éclater", roum. tocma(i) $>$ roum. dial. tomna "justement", roum. tocmi > roum. dial. tomni "arranger" (Nandris, 1963 : 152-153).

Si l'on essaie de rendre compte de ces lénitions dans un cadre binariste, nous sommes rapidement confrontés au problème de la naturalité (Brandão de Carvalho, 2008; Schane, 1984). Si la lénition est envisagée comme une perte de contenu (Lass, 1984), les traits, par leur permanence puisque seule leur valeur change, ne permettent pas de rendre compte de ce processus. Ainsi la résolution vocalique touchant $/ \mathrm{k} /$ seraient formalisée comme en (4) où, selon la métrique générative, le coût est strictement équivalent : elle ne reflète aucune perte de contenu, voire pourrait être interprétée comme un gain en complexité (dans le passage d'une valeur négative à une valeur positive).

(4) Résolution vocalique de /k/ dans un cadre binaire

$\left.\begin{array}{l}\text { +consonantique } \\ \text {-vocalique } \\ \text {-coronal } \\ \text {-continu } \\ \text {-sonant }\end{array}\right] \rightarrow\left(\begin{array}{l}\text {-consonantique } \\ \text {-vocalique } \\ \text {-coronal } \\ + \text { continu } \\ + \text { sonant }\end{array}\right)$

Afin de résoudre ces difficultés, nous proposons des représentations basées sur les éléments (KLV, 1988; Angoujard, 1997; Scheer, 1999; Backley, 2011) dans le cadre de la phonologie du gouvernment (KLV, 1990; Lowenstamm, 1996; Scheer, 2004). Cependant, nous verrons que la hiérarchie élémentaire que nous visons ne pourra être une traduction directe de la hiérarchie proposée en Fig. 3. De plus, nous montrerons que les résultats obtenus grâce à la hiérarchie unaire ne peuvent être considérés indépendamment de la structure syllabique.

\title{
4.2 Unarisme vs binarisme et représentation élémentaire
}

Le passage des traits aux éléments pourrait signifier une simple substitution d'objets binaires par des objets unaires. Une telle approche ne serait pas sans précédent et a déjà été envisagée, notamment par Angoujard (2006 : 39), qui adapte la géométrie des traits de Clements (1985) aux éléments :

Figure 5 : Géométrie des éléments (Angoujard, 2006)

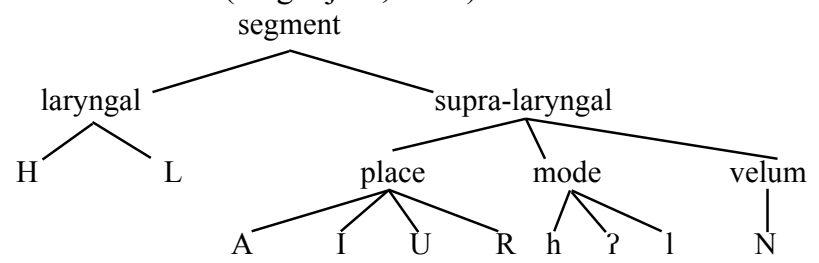

En effet, cette adaptation est tout à fait envisageable si l'on considère, à l'instar de Harris (1994), que tout élément renvoie nécessairement à une propriété phonétique. Toutefois, la structure en Fig. 5 ne saurait être compatible avec l'approche contrastiviste, notamment car elle n'a pas pour vocation de faire émerger les contrastes, mais de représenter toutes les propriétés des sons. Cette approche des éléments s'inscrit dans ce que Jensen (1994) a nommé néo-segmentaliste : c'est-à-dire une approche où les éléments sont de simples substituts des traits binaires qui reconduisent l'analyse antérieure au cadre autosegmental ${ }^{11}$. On 
en voit rapidement les limites dans le cas qui nous préoccupe. Si l'on applique la même démarche et que l'on combine la théorie des éléments avec la structure de la hiérarchie présentée précédemment en Fig. 3, on obtient la représentation suivante en Fig. 6. C'est-à-dire, un arbre dont les nœuds sont occupés, non plus par des traits, mais par des matrices d'éléments ${ }^{12}$ :

Figure 6 : Traduction de la hiérarchie binaire en éléments

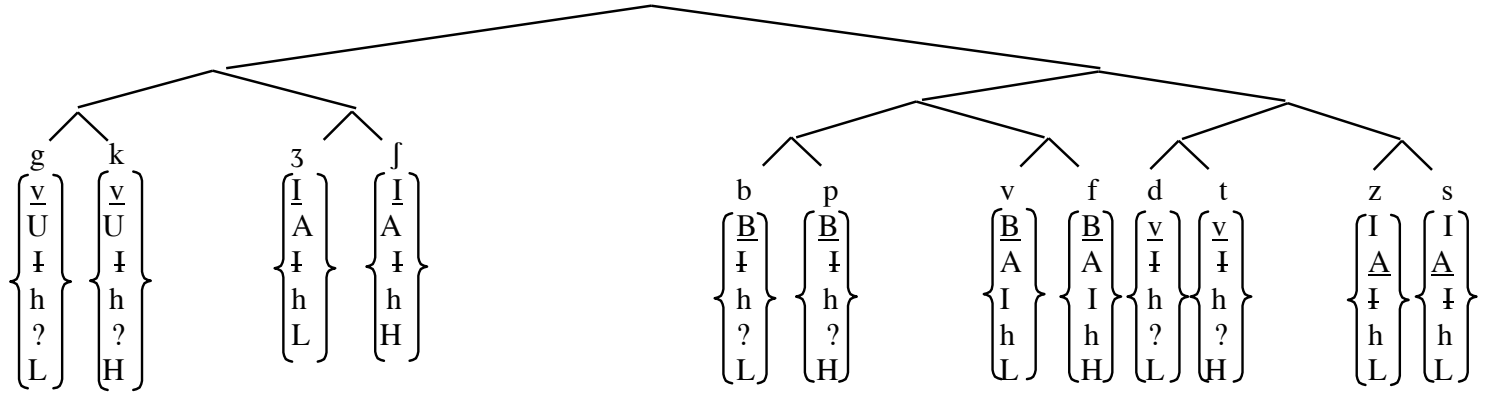

Cette représentation élémentaire, si nous la maintenons telle quelle, s'avère problématique pour plusieurs raisons.

L'architecture globale des hiérarchies contrastives s'obtient par le biais de bifurcations - chaque nœud correspond à un trait et se divise en deux branches, une pour chaque valeur.

Au-delà du problème que pose l'utilisation d'objets privatifs dans une structure conçue pour des primitives binaires, s'il fallait maintenir la précédente architecture binaire pour une future hiérarchie unaire, on ne saurait justifier l'organisation de ces nœuds. La pertinence des sous-groupes en Fig. 6 (/g, k, J, 3/, /p, b, v, f/, /d, t, z, s/) dépend de ce que Basbøll (2005 : 103) appelle les sous-systèmes intégrés. Cette notion part de l'idée que les segments partageant une majorité de leur contenu forment un sous-système au sein du système global de la langue. Alors que l'on peut aisément justifier les relations entretenues par les segments au sein de chaque sous-sytème (puisqu'il s'agit des classes naturelles), l'organisation des soussystèmes entre eux paraît plus arbitraire. En effet, sur la base de quoi, peut-on, en français justifier que le nœud dominant les labiales est relié au nœud dominant les coronales par opposition au nœud dominant les vélaires?

Afin de montrer plus précisément en quoi l'organisation des sous-systèmes en Fig. 6 ne répond pas particulièrement aux faits observés dans la phonologie du français, nous revenons sur deux phénomènes s'étant déroulé diachroniquement : la fusion de la labiale et de jod en fricative palatale (lat. sapiam $>$ sache) et la réalisation de $/ \mathrm{k} /$ en occlusive coronale (placere $>$ IIIèmes. plakJere, plajtsere $>$ IVème $2 \mathrm{~s}$. plajdzere $>$ VIIèmes. plaidzir > plaisir). Alors que le premier phénomène nous indique une relation particulière entre les labiales et les vélaires/palatales et qui ne concerne pas les coronales, le second phénomène met en avant la relation entretenue entre vélaires et coronales, excluant les labiales. De fait, puisqu'il doit nécessairement y avoir adéquation entre la hiérarchie contrastive et la phénoménologie qu'elle explique et que l'organisation des sous-ensembles n'a pas de raison d'être autre que de permettre à la hiérarchie de justifier les phénomènes, il faudrait accepter de modifier cette organisation pour représenter, avec le plus de justesse, les phénomènes consonantiques. Cela impliquerait d'avoir plusieurs hiérarchies pour rendre compte d'une même langue. En conséquence, les représentations en Fig. 7 (a) et (b) sont tout à fait envisageables pour le français. Cependant, une telle permissivité serait en désaccord avec la démarche contrastiviste.

Figure 7 : Organisations possibles des sous-systèmes intégrés

(a)

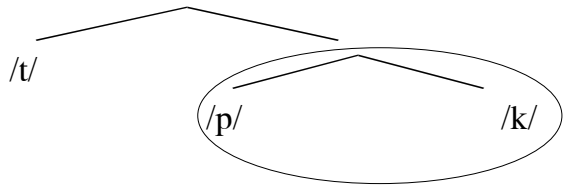


(b)

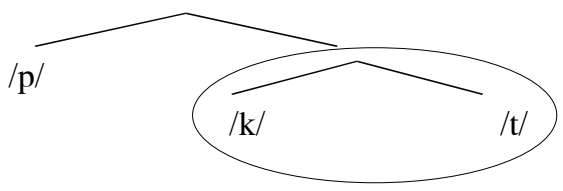

\subsection{Hiérarchie}

Afin de développer une hiérarchie unaire pertinente et cohérente avec les remarques faites dans la soussection précédente, nous n'utilisons pas les éléments comme les traductions des traits. En effet, contrairement aux traits, les éléments offrent plus de plasticité et permettent de se détacher de la "réalité" phonétique. C'est donc cet aspect que nous proposons de développer dans une hiérarchie qui manipule deux éléments : $<\mathrm{I}>$ pour [coronal] et $<\mathrm{U}>$ pour [labial]. Ces éléments sont les équivalents de [aigu] et [grave], respectivement, dans la perspective jakobsonienne. La phénoménologie précédente, où les dorsales et les palatales s'affaiblissent en labiales ou coronales, nous conduit à considérer que cette classe [compact] contient, par le Principe de Projection Elargi (Scheer, 1996) les deux dimensions (aigu et grave). En d'autres termes, pour représenter les trois lieux d'articulation du français, la structure en Fig. 8 suivante ne nécessite que deux primitives segmentales et deux types de relations (ET et OU).

Figure 8 : Hiérarchie privative

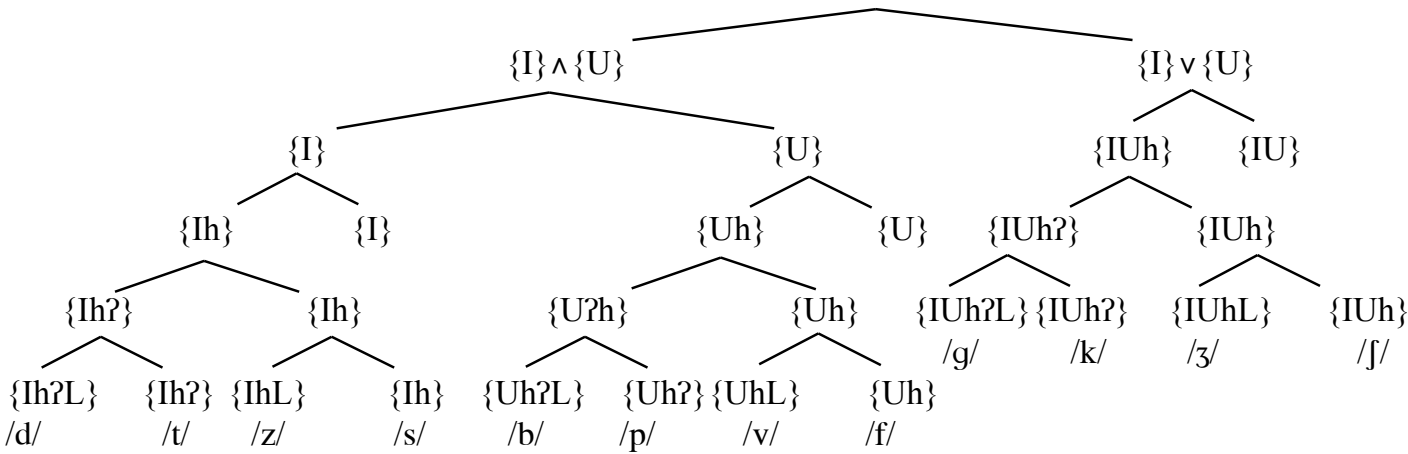

La première bifurcation de la structure hiérarchique en Fig. 8 propose soit la conjonction, soit la disjonction des élément $\{\mathrm{I}\}$ et $\{\mathrm{U}\}$. On a alors dans la partie gauche de l'arbre, les segments contenant soit $\{I\}$, soit $\{U\}$, respectivement les coronales et les labiales, et dans la partie droite, les segments contenant à la fois $\{\mathrm{I}\}$ et $\{\mathrm{U}\}$, autrement dit les vélaires. Ces éléments, parce qu'ils sont essentiels dans la représentation des segments, sont ceux qui apparaissent en premier. Les autres éléments, ceux servant à qualifier l'articulation des consonnes, sont introduits progressivement.

Le second niveau de bifurcation introduit l'élément de bruit $\{h\}$, présent dans toutes les consonnes, sauf les semi-voyelles. Ce palier permet alors de distinguer $/ \mathrm{j} /$ et $/ \mathrm{w} / \mathrm{du}$ reste des consonnes.

Au troisième palier apparaît l'élément $\{$ P $\}$. C'est à ce niveau que ressortent les fricatives $(/ \mathrm{z}, \mathrm{s} /, / \mathrm{v}, \mathrm{f} / \mathrm{et} / 3$, J/) qui ne sont pas concernées par l'occlusion.

La toute dernière bifurcation permet l'introduction de l'élément $\{\mathrm{L}\}$, servant à marquer le voisement, hautement contrastif parmi les obstruantes du français. Ainsi, on peut distinguer entre /p, t, k/ et /b, d, g/ d'une part et entre /s, f, J/ et/z, v, 3/ d'autre part.

Il est à noter que la representation donnée en Fig. 8 est en corrélation avec la proposition de Jakobson (1969) concernant l'acquisition du système phonologique: acquérir un système adulte, c'est acquérir du contraste. En d'autres termes, il s'agit de compléxifier la structure initiale (partie supérieure de la hiérarchie en Fig. 8 par ajout de strates, jusqu'à ce que tous les phonèmes de la langue soient présents. Ainsi, plus les segments apparaissent bas dans la hiérarchie, plus ils sont complexes. 
Le problème de naturalité évoqué précédemment dans la section 4.1. s'en trouve ainsi résolu : en effet, nous avons mis en avant le fait que la lénition, mécanisme impliquant une perte de contenu pour le segment touché, n'était pas fidèlement reflétée par l'utilisation des traits binaires. La représentation du système que nous proposons en Fig. 8 permet, en revanche, d'illustrer directement la perte de contenu : pour faire surface, une consonne affaiblie prend la réalisation d'un segment situé au dessus d'elle dans la hiérarchie. Ce segment est, par définition, moins complexe. Si l'on reprend le cas de la spirantisation de l'occlusive sonore /b/ qui a eu lieu dans la diachronie du français (voir Tableau 5), et que l'on l'illustre avec les matrices issues de notre hiérarchie unaire, on peut désormais mieux apprécier la perte de contenu :

(5) Spirantisation de /b/ du latin au français habere $>\mathrm{I}^{\mathrm{er}}$ s. haßere $>\mathrm{III}^{\mathrm{ème} 2}$ s. avere $>\mathrm{XVIII}^{\mathrm{e}}{ }^{\text {èe2 }}$ s avoir

$\begin{array}{ccc}/ \mathrm{b} / & \rightarrow & {[\mathrm{v}]} \\ \{\mathrm{UPhH}\} & \{\mathrm{UhH}\}\end{array}$

Pour avoir une réalisation continue, l'occlusive perd tout simplement son élément d'occlusion - dans la hiérarchie en Fig. 8, cela revient à élaguer la branche au bout de laquelle se trouve /b/ de façon à ce que $\mathrm{seul} / \mathrm{v} /$ soit accessible.

De manière analogue, le cas de la résolution vocalique de $/ \mathrm{k} / \mathrm{s}$ 'explique par perte d'un élément de lieu, ainsi que des éléments de mode :

(6) Résolution vocalique de $/ \mathrm{k} /$ dans un cadre unaire :

$$
\left\{\begin{array}{l}
\mathrm{l} / \\
\mathrm{IU} \\
\mathrm{P} \\
\mathrm{h}
\end{array}\right\} \quad \rightarrow \quad \begin{array}{lll}
/ \mathrm{j} / & / \mathrm{w} / \\
\mathrm{I}\} & \{\mathrm{U}\}
\end{array}
$$

Le cas de la résolution vocalique est parfaitement similaire aux cas de léntion aboutissant à une autre classe comme il en va pour le roumain mais aussi pour l'albanais alb. luftë $<$ lat. lucta, alb. troftë $<$ lat. tructa, alb. ftua $<$ lat. cotoneum (Sala, $1970: 172$ ) et bien entendu pour le français lat. surgere, fulgur $>$ sourdre, foudre. Ici, seul le lieu change par perte d'un élément $(<\mathrm{I}>$ ou $<\mathrm{U}>)$, le mode changeant uniquement en albanais (par perte de l'élement occlusif).

\section{Conclusion}

Nous avons montré que l'approche contrastive, telle qu'elle est pratiquée dans les modèles binaristes ne permet pas de saisir la complexité des processus qui s'appuient sur la syllabe. D'autre part, nous avons montré que les démarches classiquement proposées pour rendre compte de la structure du système des obstruantes du français échouent à justifier la place, et de fait la structure interne, occupée par les segments. Nous avons proposé, en conséquence, de réviser ces propositions en nous appuyant sur les faits diachroniques. Seule une représentation élémentaire est à même de rendre compte de façon transparente des phénomènes de lénitions. La hiérarchie que nous avons formulée suppose la remise en question des représentations élémentaires admises jusqu'à présent. Ces dernières ne permettent pas de comprendre les principes en jeu dans la lénition : le lien entre la consonne affaiblie et son reflexe est complètement absent de la représentation. Notre proposition, en intégrant le contraste, permet de retrouver ce lien parce qu'elle considère que les éléments constitutifs des segments lénifiés font déjà partie de la structure de la consonne originelle. La lénition, dans cette perspective, ne résulte que de l'élagage de la hiérarchie.

L'application de la méthode contrastiviste n'a pas de pertinence sans intégration de la dimension syllabique. L'utilisation des primitives $\{\mathrm{I}\}$ et $\{\mathrm{U}\}$ et les opérations sur ces éléments, constitue un pas vers cette intégration et s'inscrit dans une dynamique qui permettra, à terme, de réduire l'inventaire des éléments nécessaires à la caractérisation des segments (Pöchtrager \& Zivanovic, 2010 ; Jensen, 1994 ; Brandão de Carvalho, 2008) 


\section{Références bibliographiques}

Angoujard J-P. (1997). Théorie de la syllabe. Rythme et qualité. Paris : CNRS Editions.

Angoujard J-P. (2006). Phonologie déclarative. Paris : CNRS Editions.

Backley P. (2011). An Introduction to Element Theory. Edinburgh University Press.

Basbøll H. (2005). Phonology of Danish. New York : Oxford University Press.

Bourciez, E. \& Bourciez, J. (1967). Phonétique française : Etude historique. Paris : Klincksieck.

Brun-Trigaud, G. \& Scheer T. (2007). La lénition des attaques branchantes en français et dans les dialectes de l'ALF, in Barra-Jover M., Brun-Trigaud G., Dalbera J-P, Sauzet P. \& Scheer T., Approches de la variation linguistique gallo- romane.

Carvalho J. Brandão de (2002). De la syllabation en termes de contours CV. Mémoire d'Habilitation à Diriger des Recherches. EHESS.

Carvalho J. Brandão de (2008). From Positions to Transitions : A Contour-Based Account of Lenition, in Carvalho J. Brandão de, Scheer T. \& Ségéral P., Lenition and fortition. Berlin : Mouton de Gruyter : 415 - 445.

Clements G. N. (1985). The Geometry of Phonological Features, in Phonology Yearbook 2, 225-252

Clements G. N. (2003). Feature Economy in Sound Systems, in Ewen C. J. \& Kaisse E. M., Phonology 20,287 334.

Dart S. (1991). Articulatory and Acoustic Properties of Apical and Laminal Articuations, UCLA Working Papers in Phonetics 79. Los Angeles: UCLA Phonetics Laboratory.

Dresher B. E. (2007). Variability in Trubetzkoy's Classification of Phonological Oppositions, The LACUS Forum 33, $133-142$.

Dresher B. E. (2009). The Contrastive Hierarchy in Phonology. Ms.

Dresher B. E. (2014). Contrastive Hierarchies in Element Theory. Conférence Theoretical Issues in Contemporary Phonology: Reading Tobias Scheer. EHESS : Paris.

Dresher B. E., Harvey C. \& Oxford W. (2013). Synchronic systems in Diachronic Change: The Role of Change. Présenté à la $36^{\text {ème }}$ rencontre Generative Linguistics in the Old World, Lund.

Elbert S. H. \& Pukui M. K. (1979). Hawaiian Grammar. The University Press of Hawaii.

Goldsmith J. A. (1990). Autosegmental and Metrical Phonology. Cambridge Mass.: Blackwell.

Hall D. C. (2007). The role and Representation of Contrast in Phonological Theory. Thèse de doctorat, Université de Toronto. Ms.

Harris J. (1994). English Sound Strucure. Oxford - Cambridge : Blackwell.

Hockett C. F. (1955). A manual of phonology. Baltimore : Waverly Press.

Jakobson R. \& Lotz J. (1949). Notes on the French Phonemic Pattern, Word 5, 151 - 157.

Jakobson R. (1969). Langage enfantin et aphasie. Paris : Éditions de minuit.

Jakobson R. (1984). Une Vie dans le langage, Autoportrait d'un savant. Paris : Éditions de Minuit.

Jensen S. (1994). Is $?$ an Element? Towards a Non-segmental Phonology, SOAS Working Papers in Linguistics and Phonetics 4, $71-78$.

Kaye J. Lowenstamm J. \& Vergnaud J. R. (1988). La structure interne des éléments phonologiques : une théorie du charme et du gouvernement, Recherches Linguistiques de Vincennes 17, 109- 134.

Kaye J. Lowenstamm J. \& Vergnaud J. R. (1990). Constituent Structure and Government in Phonology, Phonology 7, $193-231$.

Lass R. (1984). Phonology. An Introduction to basic Concepts. Cambridge University Press.

Lowenstamm J. (1996). CV as the only syllable type, in Durand J. \& Laks B., Current trends in phonology: Models and Methods. Salford - Manchester : ESRI, $419-441$.

Martinet A. (1970). Eléments de linguistique générale. Paris : Armand Colin.

Martinet A. (1965). Linguistique synchronique. Etudes et recherche. Paris : Presses Universitaires de France.

Nandris O. (1963). Phonétique historique du roumain. Paris : Klincksieck.

Pöchtrager M. A. \& Zivanovic S. (2010). GP 2 and Putonghua too, Acta Linguistica Hungarica 57(4) : 357 - 380.

Sala M. (1970). Conributii la fonetica istorica a limbii române. tr. fr. : Contributions à la phonétique historique du roumain, (1976). Paris : Klincksieck.

Schane A. S. (1984). The Fundamentals of Particle Phonology, in Euren C. \& Anderson J., Phonology Yearbook, volume I. Cambridge University Press, 129 - 155.

Scheer T. (1996). Une théorie de l'interaction entre consonnes. Contribution au modèle syllabique CVCV, alternances e-ø dans les préfixes tchèques, structure interne des consonnes et la théorie X-barre en phonologie. Thèse de doctorat, Université Paris 7. Ms.

Scheer T. (1999). A Theory of Consonantal Interaction, Folia Linguistica 32, 201 - 237.

Scheer T. (2004). A lateral theory of phonology. Vol 1: What is CVCV, and why should it be ?. Berlin : de Gruyter. 
Scheer T. (2010). How to marry (structuralist) contrast and (generative) processing (review of Dresher 2009, The Contrastive Hierarchy in Phonology). Lingua 120: 2522-2534.

Scheer T. \& Ségéral P. (2001). La coda-miroir, Bulletin de la Société de Linguistique de Paris 96, 107 - 153.

Stevens K. N. \& Keyser S. J. (2006). Enhancement and Overlap in the Speech Chain, Language 82, 33 - 63. Troubetzkoy S. N. (1939). Grundzüge der Phonologie. tr. fr : Principes de phonologie. Paris : Klincksieck. Zink, G._(1986). Phonétique historique du français. Paris : PUF.

\footnotetext{
${ }^{1}$ Voir notamment le débat Dresher (2014), Scheer (2010) qui porte sur la possibilité d'appliquer, ou non, l'hypothèse contrastiviste à la théorie des éléments.

${ }^{2}$ Un relecteur anonyme souligne que " phénoménologie diachronique du français » paraît trop général. Nous verrons que, si l'interaction vélaires/palatales est, ici, traitée prioritairement c'est que les autres lieux d'articulation ne posent pas problème dans les cadres phonologiques actuels. On s'accorde à considérer que les lieux [labial] et [coronal] recouvrent en français l'ensemble des obstruantes labiales et labiodentales, dentales et alvéolaires respectivement. Ce qui, en revanche, est problématique, c'est l'absence de lieu couvrant les occlusives vélaires ET les fricatives palatales.

${ }^{3}$ Le cadre syllabique, même si il ne peut être développé en détail dans cet article, est indissociable de la représentation élémentaire (voir par exemple, KLV : 1985, Angoujard : 1997, Harris : 1994). La réduction du nombre de primitives élémentaires, qui sous-tend notre proposition, s'accompagne nécessairement d'une structure hôte à même de prendre en charge le rôle traditionnellement dévolu aux traits/éléments, en particulier laryngaux (Carvalho : 2002, 2008, Jensen : 1994, Pöchtrager : 2006, Pöchtrager \& Zivanovic : 2012).
}

${ }^{4}$ Notons cependant que, dans le cadre du phonetic enhancement (Stevens \& Keyser : 2006), les traits a priori redondants peuvent toutefois être utiles au contraste (Dresher 2009 : 273).

${ }^{5}$ Concernant la discussion de la réalité des traits articulatoires contre l'abstraction des traits acoustiques, nous renvoyons à l'étude de Dart (1991 : 48 et sq.) qui montre, palatalogrammes et linguagrammes à l'appui, que les segments classiquement associé à l'étiquette articulatoire dental présentent une énorme variation. Par exemple, 42\% des réalisations de $/ \mathrm{t}, \mathrm{d}, \mathrm{n} /$ ne sont pas dentales mais alvéolaires, ou encore, $/ 1 /$, systématiquement considéré comme une dentale et donc associé à la classe précédente, est majoritairement alvéolaire ou postalvéolaire. Ces variations sont encore plus flagrantes si l'on se réfère à l'articulateur mobile (apical, apicolaminal, laminal). Pour Backley (2011: 90) : «It appears that phonetic labelling can be more abstract than we might think ».

${ }^{6}$ Voir Dresher (2007), en particulier pour une discussion sur cette impossibilité en français, à la différence du système des obstruantes du grec où l'alignement serait le seul possible.

${ }^{7}$ Dans le cas du rapport palatales / vélaires, la stridence n'est pas que simple ouverture du canal, elle est renforcée par l'avancement du point d'articulation. Cf. Stevens \& Keyser (2006).

${ }^{8}$ Hockett (1955 : 173), "[b ]oth of these decompositions of the French obstruents have the odor of pure game-playing, an odor which is seemingly appetizing to some linguists".

${ }^{9}$ Pour la position $\{-r\}$, qui ne sera pas abordée ici, voir Brun-Trigaud \& Scheer (2007) en particulier pour ses conséquences au niveau syllabique.

${ }^{10}$ Zink (1986 : 65).

${ }^{11}$ Concernant l'Absolute Slicing Hypothesis voir Goldsmith (1990).

${ }^{12}$ Nous utilisons, dans cette première structure, les matrices élémentaires proposées par Scheer (1999). Il faut noter que les propositions de Harris (1994) et Angoujard (1997) susciteraient les mêmes remarques quant à la naturalité et à la possibilité de rendre compte de la phénoménologie des obstruantes du français. 